\title{
openheart Determinants of aortic growth rate in patients with bicuspid aortic valve by cardiovascular magnetic resonance
}

\author{
Froso Sophocleous, ${ }^{1}$ Bostjan Berlot (10 ,1,2 Maria Victoria Ordonez, ${ }^{1}$ \\ Mai Baquedano, ${ }^{1}$ Elena Giulia Milano, ${ }^{3,4}$ Viola De Francesco, ${ }^{2}$ Graham Stuart, ${ }^{2}$ \\ Massimo Caputo, ${ }^{1,2}$ Chiara Bucciarelli-Ducci, ${ }^{1,2}$ Giovanni Biglino (D) 1,2,5
}

\begin{abstract}
- Additional material is published online only. To view please visit the journal online (http://dx.doi.org/10.1136/ openhrt-2019-001095).
\end{abstract}

To cite: Sophocleous F, Berlot B Ordonez MV, et al. Determinants of aortic growth rate in patients with bicuspid aortic valve by cardiovascular magnetic resonance. Open Heart 2019;6:e001095. doi:10.1136/ openhrt-2019-001095

Received 22 May 2019 Revised 19 July 2019 Accepted 12 September 2019

A) Check for updates

(C) Author(s) (or their employer(s)) 2019. Re-use permitted under CC BY. Published by BMJ.

${ }^{1}$ Bristol Medical School, University of Bristol, Bristol, UK ${ }^{2}$ Bristol Heart Institute, University Hospitals Bristol NHS Foundation Trust, Bristol, UK

${ }^{3}$ Institute of Cardiovascular Science, University College London, London, UK ${ }^{4}$ Department of Surgery, Dentistry, Paediatrics and Gynaecology, University of Verona, Verona, Italy ${ }^{5}$ National Heart and Lung Institute, Imperial College London, London, UK

Correspondence to Dr Giovanni Biglino; g.biglino@ bristol.ac.uk

\section{ABSTRACT}

Objectives This study aimed to identify determinants of aortic growth rate in bicuspid aortic valve (BAV) patients. We hypothesised that (1) BAV patients with repaired coarctation (CoA) exhibit decreased aortic growth rate, (2) moderate/severe re-coarctation (reCoA) results in increased growth rate, (3) patients with right non-coronary (RN) valve cusps fusion pattern exhibit increased aortic growth rate compared with right-left cusps fusion and type 0 valves.

Methods Starting from $n=521 B A V$ patients with cardiovascular magnetic resonance data, we identified $n=145$ patients with at least two scans for aortic growth analysis. Indexed areas of the sinuses of Valsalva and ascending aorta ( $A A 0)$ were calculated from cine images in end-systole and end-diastole. Patients were classified based on dilation phenotype, presence of $\mathrm{CoA}$, aortic valve function and BAV morphotype. Comparisons between groups were performed. Linear regression was carried out to identify associations between risk factors and aortic growth rate.

Results Patients ( $39 \pm 16$ years of age, $68 \%$ male) had scans $3.7 \pm 1.8$ years apart; 32 presented with $A$ Ao dilation, 18 with aortic root dilation and 32 were overall dilated. Patients with repaired $\mathrm{CoA}(\mathrm{n}=61)$ showed decreased aortic root growth rate compared with patients without CoA $(p \leq 0.03)$ regardless of sex or age. ReCoA, aortic stenosis, regurgitation and history of hypertension were not associated with growth rate. RN fusion pattern showed the highest aortic root growth rate and type 0 the smallest ( 0.30 vs $0.08 \mathrm{~cm}^{2} / \mathrm{m}^{\star}$ year, end-systole, $p=0.03$ ).

Conclusions Presence of CoA and cusp fusion morphotype were associated with changes in rate of root dilation in our BAV population.

\section{INTRODUCTION}

Bicuspid aortic valve (BAV) is a highly heterogeneous congenital heart disease (CHD), commonly associated with increased risk of developing thoracic aortic aneurysms and acute aortic dissection, ${ }^{1}$ the latter increasing after aortic valve replacement (AVR). ${ }^{2}$ BAV aortopathy can range from slow-asymptomatic aortic diameter growth to rapid progression or early-life threatening

\section{Key questions}

What is already known about this subject?

- Patients with bicuspid aortic valve (BAV) present with different degrees of aortic dilation. Despite known phenotypic variations in BAV aortopathy, and differences in aortic architecture in BAV patients with concomitant aortic coarctation (CoA), determinants of aortic growth rate are not fully known.

What does this study add?

- This study highlights differences in BAV patients with/without CoA based on cardiovascular magnetic resonance imaging data, identifying particularly differences in root dilation. The study also reinforces previous echocardiographic observations on the association between BAV morphotype and differences in aortic dilation, using a different imaging modality.

How might this impact on clinical practice?

- This study suggests that CoA patients with concomitant BAV disease could be treated as a separate group with a different phenotype, thus refining risk stratification and monitoring of potential progression of BAV aortopathy.

aortic complications. ${ }^{3}$ In vitro and in vivo studies have explored the inherent defect of aortas in BAV patients leading to altered wall mechanical properties which contribute to aneurysm formation. ${ }^{45}$ However, at present there is no established risk marker to help in the prognosis of BAV aortic growth. ${ }^{6}$ Known predictors of BAV aortopathy progression include older age, male sex, increased systolic blood pressure, valvular dysfunction and BAV morphology. ${ }^{78}$ While no consistent correlation has been reported between BAV morphotype and aortopathy pattern, ${ }^{9}$ the phenotype of dilation is reported to be predictive of the disease course, and thus being used to facilitate risk stratification and standardise surgical approaches. ${ }^{1011}$

$\mathrm{BAV}$ is often concomitant with aortic coarctation $(\mathrm{CoA})^{12}$ and aortic wall complications 
are frequent in adults with $\mathrm{CoA},{ }^{13}$ whereas those with progressive course of the disease may also develop aortic dilation and be at risk of aneurysm and aortic dissection. Recent studies have shown that ascending and post-CoA aortic diameters or dilations are linked to the degree of CoA severity ${ }^{14}$ and that, in a CoA population, patients with BAV were more likely to have moderate or severe ascending aorta (AAo) dilation compared with those with a trileaflet valve. ${ }^{15}$ While BAV-aortopathy is likely a reflection of BAV morphotype rather than CoA or its physiological effects, ${ }^{16}$ questions remain about changes in aortic growth in $\mathrm{BAV}+\mathrm{CoA}$ patients compared with isolated BAV. Furthermore, the coexistence of CoA with BAV has been associated with increased risk of aortic dissection compared with patients with isolated $\mathrm{CoA},{ }^{17}$ but does aortic growth rate vary compared with patients with isolated BAV?

Taking advantage of a large cohort of BAV patients with and without CoA, this study aims to assess the effect of $\mathrm{CoA}$ and other potential determinants on aortic growth rate. Based on a recent study from our group reporting morphological differences in the aortic arch architecture in BAV patients with/without $\mathrm{CoA},{ }^{18}$ the primary hypothesis is that $\mathrm{BAV}+\mathrm{CoA}$ patients exhibit slower aortic growth rate, while patients with re-coarctation (reCoA) exhibit increased aortic growth rate. Furthermore, based on differences in aortic growth across BAV fusion types established with echocardiography, ${ }^{19}$ a secondary hypothesis is that patients with right non-coronary $(\mathrm{RN})$ valve fusion type exhibit increased aortic growth rate.

\section{METHODS \\ Patient population}

This was a longitudinal single-centre retrospective study. Consecutive BAV patients $(n=521)$ were identified in the cardiovascular magnetic resonance (CMR) imaging database between 2009 and 2017, of which 182 patients had CMR scans at two or more time-points before AVR. When patients had more than two scans available, the least and the most recent were considered. Exclusion criteria included: suboptimal image quality $(n=7)$, unconfirmed bicuspid morphology $(\mathrm{n}=6$, even after consulting transthoracic/trans-oesophageal echocardiography or CT angiography when in doubt), degenerative aortic valve $(n=1)$, concomitant either moderate or complex CHD ( $n=2$ Shone's complex, $n=2$ Tetralogy of Fallot and $n=1$ Epstein's anomaly), connective tissue disorders $(\mathrm{n}=1$ Marfan, $\mathrm{n}=10$ Turner and $\mathrm{n}=1$ Ehlers Danlos syndromes, confirmed by genetic results), other diseases ( $n=1$ pseudo-CoA, n=1 Kawasaki disease), unrepaired CoA ( $n=6)$ and surgeries structurally affecting the aortic valve and/ or the aorta $(n=1$ aortic valvotomy and $n=1$ aortic arch reconstruction). A flowchart is provided in figure 1 . All CMR data were acquired at $1.5 \mathrm{~T}$ (Avanto, Siemens Healthineers, Erlangen, Germany). Demographic and functional variables were collected from CMR reports, including age, height, sex, aortic valve morphology,

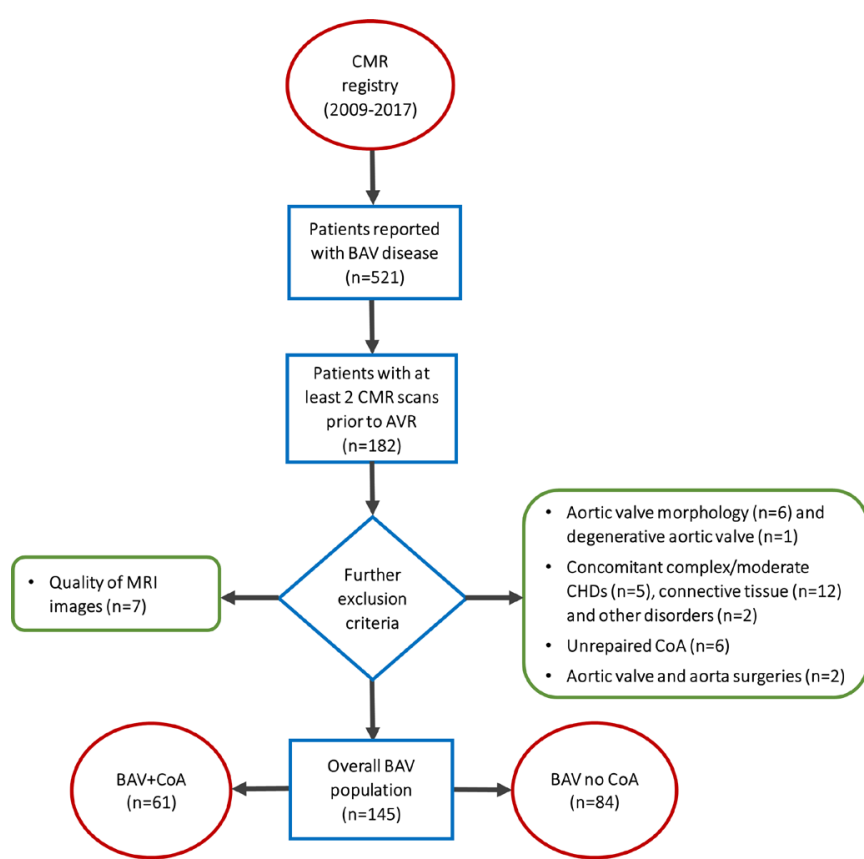

Figure 1 Patient selection. Inclusion criteria: two CMR scans prior to AVR $(n=182)$. Exclusion criteria: suboptimal quality of CMR cine images $(n=7)$, unconfirmed bicuspid morphology $(n=6)$, degenerative aortic valve, any concomitant either complex or moderate CHDs, including Shone's complex $(n=2)$, tetralogy of Fallot $(n=2)$ and Epstein's anomaly $(n=1)$; connective tissue disorders, including Marfan $(n=1)$, Turner $(n=10)$ and Ehlers Danlos $(n=1)$ syndromes; pseudo-CoA $(n=1)$, Kawasaki disease $(n=1)$, unrepaired $\operatorname{CoA}(n=6)$ and surgeries $(n=2)$ such as aortic valvotomy and aortic arch reconstruction. AVR, aortic valve replacement; $\mathrm{BAV}$, bicuspid aortic valve; CMR, cardiovascular magnetic resonance; $\mathrm{CHD}$, congenital heart disease.

severity of aortic regurgitation or stenosis, presence of repaired CoA, degree of reCoA and history of hypertension. The population was then divided into two subgroups, that is, patients with isolated BAV and patients with BAV and repaired CoA.

\section{Aortic measurements}

The aortic measurements were performed in the steadystate free precession cine images acquired in the threechamber (3C) and left ventricular outflow tract (LVOT) views and in the oblique sagittal views, both at the level of the sinuses of Valsalva $(\mathrm{SoV})$ and at the proximal AAo (figure 2). The axial views of the AAo were acquired using multiple planes resulting in true perpendicular planes, to consider possible dynamic changes over time and possible asymmetry of the aorta in keeping with the 2017 European Society of Cardiology Guidelines on Valvular Heart Disease. ${ }^{20}$ The AAo measurement was taken at the level of the right pulmonary artery. Having verified intraobserver and inter-observer reproducibility on 20 random cases (intraclass correlation coefficient $=0.988$ and 0.967 , respectively), all measurements were taken by a single operator, who received the anonymised cases in random sequence and was blinded as to the history of CoA. Aortic 

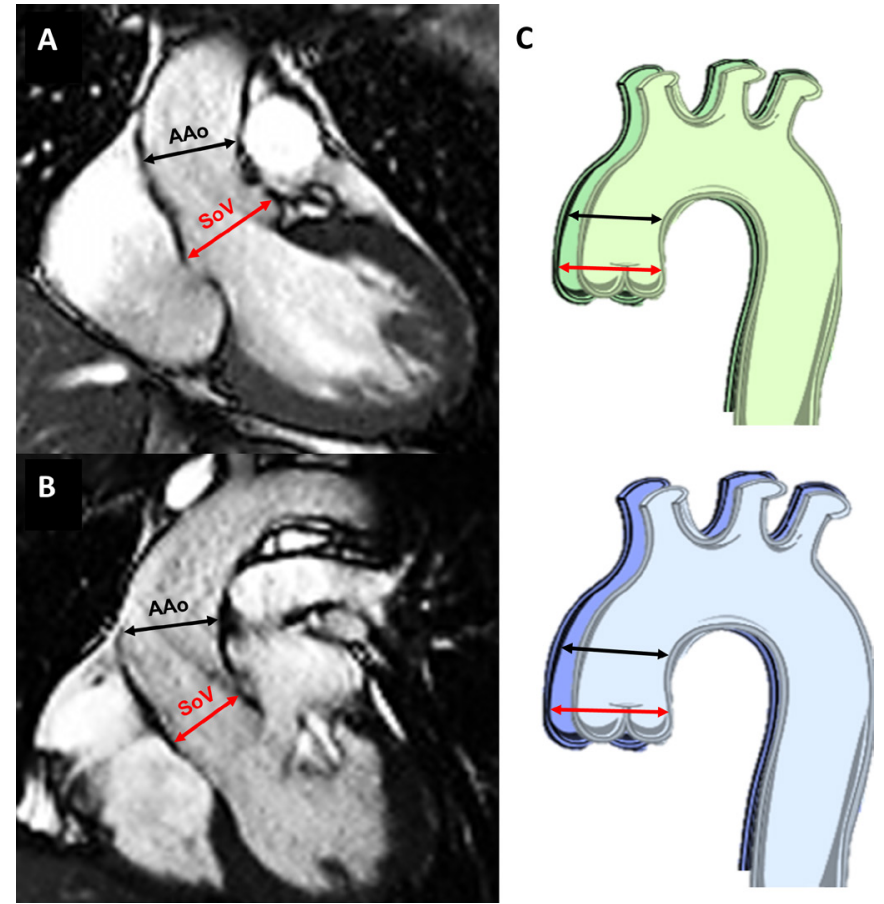

Figure 2 CMR measurements. Cine images showing measurements of aortic diameters, $(A)$ at $3 C$ and $(B)$ at left ventricular outflow tract view. The red lines show the diameters measured at the SoV level and the black lines at the proximal AAo. (C) Schematic representation of changes at end-diastole (green) and end-systole (blue) over time to capture the possible dynamic nature of dimensional changes. AAo, ascending aorta; SoV, sinuses of Valsalva.

growth rate was defined as the difference in indexed aortic area over the two time-points, having normalised aortic area by height ${ }^{21}$ :

$$
\text { Growth Rate }=\frac{\frac{\mathrm{A} 2}{\mathrm{H}}-\frac{\mathrm{A} 1}{\mathrm{H}}}{\mathrm{t} 2-\mathrm{tl}}
$$

where A, area; $\mathrm{H}$, height; $\mathrm{t}$, time. In order to avoid the assumption of circularity, the aortic radius was derived as the average of the diameters at 3C and LVOT and thus area calculated as:

$$
\mathrm{A}=\pi\left(\frac{\mathrm{D}(3 \mathrm{C})}{2} * \frac{\mathrm{D}(\mathrm{LVOT})}{2}\right)
$$

where $\mathrm{D}$, diameter.

\section{Variables and classification}

Valve morphotype was classified as 'type 0' (ie, true BAV with no raphe), 'type 1' (ie, one central raphe) and 'type 2' (ie, two central raphes). Furthermore, type 1 valves were subdivided intro right coronary and left coronary (RL) fusion patter, right coronary and RN fusion pattern and left coronary and left non-coronary (LN) fusion pattern. ${ }^{9}$ ReCoA was defined as a recurrence of narrowing at the CoA site. The severity of reCoA was assessed from the ratio of the isthmus diameter to the descending thoracic aorta, and classified as severe if $<0.55$, moderate to mild if $0.55-0.85$ and absent if $>0.85$. The severity of aortic stenosis and aortic regurgitation were extracted from CMR reports and, for the purposes of this analysis,
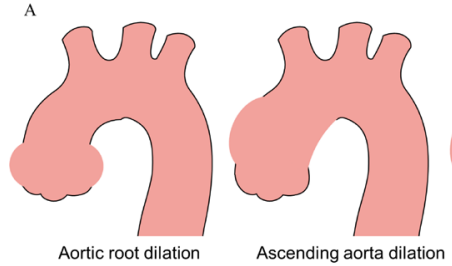

B
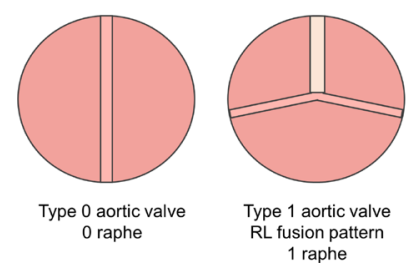
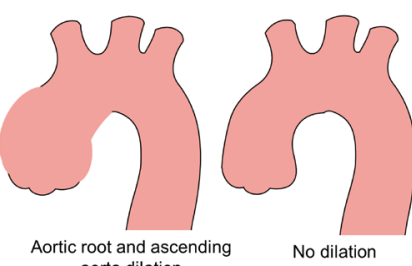

aorta dilation
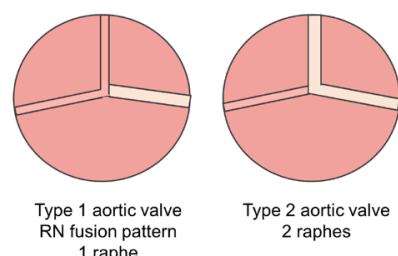
1 raphe
Figure 3 Aortic dilation phenotypes and bicuspid aortic valve (BAV) morphotypes. (A) Classification of the aortic dilation configurations in our population showing aortic root dilation, ascending aorta dilation, aortic root and ascending aorta dilation, and no dilation. (B) Classification of the BAV morphotypes showing type 0 valve with no raphe, type 1 valve with one central raphe having in one case a right coronary and left coronary fusion pattern and in the other case a right non-coronary fusion pattern, and type 2 valve with two raphes.

patients were classified as either having none-to-mild or moderate-to-severe stenosis/regurgitation. In addition, according to a modified Fazel classification of aortic dilation configurations ${ }^{1022}$ patients were classified as presenting (1) increased SoV diameter, (2) increased AAo diameter, (3) increased SoV and AAo diameters, and (4) no substantial aortic growth, as summarised in figure 3. We classified patients as 'no growth' when aortic growth rate was $<0.08 \mathrm{~cm}^{2} / \mathrm{m}^{*}$ year, corresponding to a diameter change of $<1.5 \mathrm{~mm}$ (ie, pixel spacing).

\section{Statistical analysis}

Continuous variables are presented as mean \pm SD and categorical variables as counts or proportions. Normality was assessed with Shapiro test. Continuous variables were compared with a Mann-Whitney test, or with KruskalWallis test and Dunn's test for post hoc adjustment when comparing more than two groups (eg, valve morphotypes). Categorical variables were compared with $\chi^{2}$ test. The association between demographic/clinical variables and aortic growth rate was assessed using linear regression, and only those variables showing a significant association on a univariate model were further tested in a multivariate regression model. A $p$ value $<0.05$ was considered an indicator of statistical significance, except for multiple comparisons where Bonferroni correction was applied. The analysis was carried out in R (R Foundation for Statistical Computing, Vienna, Austria).

\section{RESULTS}

A population $n=145 \mathrm{BAV}$ patients with two CMR scans

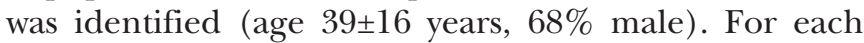
patient, the time interval between $\mathrm{CMR}$ scans was $3.7 \pm 1.8$ 


\begin{tabular}{|c|c|c|c|}
\hline $\begin{array}{l}\text { Demographic and clinical } \\
\text { variables }\end{array}$ & Repaired CoA, n=61 & NoCoA, $n=84$ & $P$ value \\
\hline Time gap between CMR scans & $4.6 \pm 1.5$ (range: $1.3-7.7$ ) & $3.0 \pm 1.9$ (range: $0.3-7.7$ ) & $<0.001^{\star \star *}$ \\
\hline Mean age at baseline & $34 \pm 13$ (range: $13-60$ ) & $42 \pm 17$ (range: $15-73$ ) & $0.006^{\star \star}$ \\
\hline Mean age at last follow-up & $38 \pm 13$ (range: $18-64)$ & $45 \pm 17$ (range: $17-77$ ) & $0.02^{*}$ \\
\hline Males & $\mathrm{n}=36,59 \%$ & $\mathrm{n}=62,74 \%$ & 0.06 \\
\hline $\mathrm{ReCOA}$ & $\mathrm{n}=33,54 \%$ & - & $\mathrm{N} / \mathrm{A}$ \\
\hline Moderate/severe reCoA & $\mathrm{n}=12,36 \%$ & - & $\mathrm{N} / \mathrm{A}$ \\
\hline Valve type 0 & $\mathrm{n}=4,7 \%$ & $\mathrm{n}=11,13 \%$ & $0.01^{*}$ \\
\hline Valve type $1, \mathrm{RL}$ fusion & $\mathrm{n}=51,84 \%$ & $n=52,62 \%$ & \\
\hline Valve type $1, \mathrm{RN}$ fusion & $\mathrm{n}=3,5 \%$ & $n=15,18 \%$ & \\
\hline Moderate/severe aortic stenosis & $\mathrm{n}=3,5 \%$ & $\mathrm{n}=26,31 \%$ & $<0.001^{\star \star \star}$ \\
\hline Moderate/severe aortic regurgitation & $\mathrm{n}=4,7 \%$ & $\mathrm{n}=19,22 \%$ & $0.009^{\star *}$ \\
\hline Hypertensive patients & $\begin{array}{l}\mathrm{n}=26,43 \% \text { ( } 22 \text { under medication, } \\
\text { three no medication and one } \\
\text { unknown) }\end{array}$ & $\begin{array}{l}\mathrm{n}=32,38 \% \text { ( } 30 \text { are under } \\
\text { medication and two unknowns) }\end{array}$ & 0.5 \\
\hline Type of CoA repair & $\begin{array}{l}\text { End-to-end }(\mathrm{n}=33) \\
\text { Subclavian flap }(\mathrm{n}=9) \\
\text { Patch }(\mathrm{n}=7) \\
\text { Stent }(\mathrm{n}=3) \\
\text { Interposition graft }(\mathrm{n}=2)\end{array}$ & & $\mathrm{N} / \mathrm{A}$ \\
\hline
\end{tabular}

CMR, cardiovascular magnetic resonance; CoA, coarctation; reCoA, re-coarctation; RL, right coronary and left coronary; RN, right noncoronary.

years (range 0.3-7.7). Also, at baseline BAV+CoA patients were significantly younger age ( $34 \pm 13$ vs $42 \pm 17, \mathrm{p}=0.006)$. Patients' characteristics are reported in table 1.

The most common BAV morphotype was the type 1 (83\%), predominantly with RL fusion type (71\%). Overall, patients presented with varying degrees of aortic stenosis (none: $\mathrm{n}=87$, mild: $\mathrm{n}=29$, moderate: $\mathrm{n}=20$, severe: $\mathrm{n}=9$ ) and aortic regurgitation (none: $\mathrm{n}=72$, mild: $\mathrm{n}=50$, moderate: $n=14$, severe: $n=9$ ), and patients with isolated BAV presented more frequently with severe stenosis and/or regurgitation (table 1). There was no difference in history of hypertension between isolated BAV and $\mathrm{BAV}+\mathrm{CoA}$ patients.

Overall, we classified 63 BAV patients without aortic dilation, 32 with AAo dilation, 18 with aortic root dilation and 32 with overall dilated aorta, table 2 . In the CoA subgroup, $54 \%$ patients had reCoA, of which $36 \%$ were moderate-to-severe.

Aortic growth rate in SoV was significantly lower in $\mathrm{BAV}+\mathrm{CoA}$ patients compared with isolated $\mathrm{BAV}$ (enddiastole: $0.05 \pm 0.09$ vs $0.13 \pm 0.2 \mathrm{~cm}^{2} / \mathrm{m} *$ year, $\mathrm{p}=0.03$; endsystole: $0.05 \pm 0.09$ vs $0.19 \pm 0.3 \mathrm{~cm}^{2} / \mathrm{m}$ *year, $\mathrm{p}=0.005$ ).

Table 2 Distribution of patients according to aortic dilation phenotype and BAV morphotype

\begin{tabular}{llcccc}
\hline End-systole & & No dilation & AAo dilation & Root dilation & Overall dilated \\
\hline CoA & Type 0 & 3 & 0 & 0 & 1 \\
& Type 1, RL & 30 & 11 & 4 & 6 \\
& Type 1, RN & 1 & 0 & 2 & 0 \\
& Type 1, LN & 2 & 1 & 0 & 0 \\
& Type 2 & 0 & 0 & 0 & 0 \\
No CoA & Type 0 & 5 & 4 & 1 & 1 \\
& Type 1, RL & 15 & 14 & 8 & 15 \\
& Type 1, RN & 4 & 2 & 3 & 6 \\
& Type 1, LN & 0 & 0 & 0 & 2 \\
& Type 2 & 3 & 0 & 0 & 32 \\
\hline \multirow{5}{*}{ Total } & 63 & 32 & $\mathbf{1 8}$ & 32
\end{tabular}

CoA, coarctation; LN, left non-coronary; RL, right coronary and left coronary; RN, right non-coronary. 
Table 3 Results of univariate linear regression analysis assessing the association of demographic/clinical variables and aortic growth rate

\begin{tabular}{|c|c|c|c|c|}
\hline \multirow[b]{2}{*}{ Demographic and clinical variables } & \multicolumn{2}{|c|}{ End-systole } & \multicolumn{2}{|c|}{ End-diastole } \\
\hline & SoV & AAo & SoV & AAo \\
\hline Age at baseline & $p=0.7$ & $p=0.7$ & $\mathrm{p}=0.9$ & $p=0.6$ \\
\hline Age at last follow-up & $p=1$ & $p=0.5$ & $p=0.8$ & $p=0.4$ \\
\hline Sex & $\mathrm{p}=0.5$ & $p=0.1$ & $\mathrm{p}=0.8$ & $\mathrm{p}=0.4$ \\
\hline Presence of $\mathrm{CoA}$ & $p=0.004^{* *}$ & $p=0.1$ & $\mathrm{p}=0.01^{*}$ & $p=0.05$ \\
\hline $\mathrm{ReCoA}$ & $p=0.8$ & $p=0.9$ & $\mathrm{p}=0.5$ & $p=0.7$ \\
\hline Severity of reCoA & $p=0.9$ & $p=0.8$ & $p=0.5$ & $p=0.4$ \\
\hline Valve morphotype & $p=0.008^{* *}$ & $p=0.07$ & $p=0.01^{*}$ & $p=0.07$ \\
\hline Severity of aortic stenosis & $p=0.8$ & $p=0.6$ & $\mathrm{p}=0.8$ & $p=1$ \\
\hline Severity of aortic regurgitation & $p=0.4$ & $\mathrm{p}=0.5$ & $p=0.3$ & $\mathrm{p}=0.7$ \\
\hline History of hypertension & $p=0.6$ & $p=0.3$ & $p=0.5$ & $\mathrm{p}=0.4$ \\
\hline
\end{tabular}

AAo, ascending aorta; CoA, coarctation; reCoA, re-coarctation; SoV, sinus of Valsalva.

Aortic growth rate in $\mathrm{SoV}$ at end-systole also varied significantly according to BAV morphotype, with RN fusion pattern showing the highest growth and type 0 the smallest $\left(0.3 \pm 0.5\right.$ vs $0.1 \pm 0.2$ vs $0.08 \pm 0.1 \mathrm{~cm}^{2} / \mathrm{m}^{*}$ year, $\mathrm{p}=0.03)$. An in-between groups analysis showed a significant difference between type $1 \mathrm{RL}$ and $\mathrm{RN}$ valves $(p=0.006)$ and between type 0 valves and type $1 \mathrm{RN}$ valves $(p=0.01)$. No correlation observed between aortic growth rate, age and sex (table 3 ).

Results from regression analysis are reported in table 3. ReCoA, severity of aortic stenosis and regurgitation, and history of hypertension were not associated with aortic growth rate, neither in the overall population nor in the BAV+CoA subgroup. In patients with CoA, type of surgical repair and age of repair did not correlate with aortic growth rate $(p \geq 0.16$ and $p \geq 0.33$, respectively) over this time window.

Multiple regression analysis showed that the concomitant absence of CoA with type 1 valve with $\mathrm{RN}$ fusion pattern in end-systole results in a $0.3 \mathrm{~cm}^{2} / \mathrm{m} *$ year increase in aortic root growth rate. From a clinical standpoint, changes in aortic diameter guide the decision to intervene, and this corresponds to $1 \mathrm{~mm} /$ year increase in aortic diameter, compared with $0.5 \mathrm{~mm} /$ year for type 1 valve with RL fusion pattern and $0.3 \mathrm{~mm} /$ year for type 0 valve.

All diameters, area values and aortic growth rate as indexed area of AAo and $\mathrm{SoV}$ both in end-systole and end-diastole, corresponding to the subgroups of CoA, NoCoA, reCoA, vale type 0 , type 1 with RL cusp fusion and $\mathrm{RN}$ cusp fusion are reported in the (online supplementary material).

\section{DISCUSSION}

This longitudinal study uses CMR data to explore changes in aortic growth rate in a cohort of BAV patients. Results showed that patients with repaired CoA tended to have slower aortic root growth rate compared with patients with isolated BAV. Previous work from our group based on a statistical shape modelling framework ${ }^{18}$ revealed nuanced differences in arch morphology in BAV patients with/without CoA, suggesting detrimental functional implications for some aortic arch architectures, and with $\mathrm{BAV}+\mathrm{CoA}$ patients generally presenting with smaller ascending and larger descending aortas compared with isolated BAV. This agrees with observations in the literature that prior $\mathrm{CoA}$ repair may protect BAV aorta from rapid dilation. ${ }^{8}$ With regards to functional implications, a recent echocardiographic study of $631 \mathrm{BAV}$ patients with and without CoA found that BAV patients with CoA were associated with increased risk of AAo complications, smaller aortic root dimensions and less severe valvular dysfunction compared with isolated BAV patients over a similar time window to that of our study, but this study did not differentiate repaired and unrepaired CoA patients. ${ }^{23}$

Our observations on growth rate, translated into yearly increase in aortic diameter, indicate that an unfavourable combination of type 1 valve with $\mathrm{RN}$ fusion in patients without CoA can result in a substantial increment in diameter of $1 \mathrm{~mm} /$ year, compared with valve type 0 where the root diameter increase was found to be $0.3 \mathrm{~mm} /$ year. Although seemingly small, these dimensions can have clinical implications, over a relatively short time window of $10-15$ years, indicating patients that potentially would require surgical intervention versus those in which the process of aortic dilation progresses at a slower rate. Similar values of aortic growth rate have been reported in the literature. AAo growth rate in BAV patients can range from 0.2 to $2.3 \mathrm{~mm}$ /year depending on patient characteristics. ${ }^{24}{ }^{25}$ According to a transthoracic echocardiogram (TTE) study performed on an isolated BAV population of 133 adults, the mean growth rate was $0.3 \mathrm{~mm}$ /year at the sinuses and $0.6 \mathrm{~mm} /$ year at the level of the AAo, whereas RL valve fusion morphotype and AAo dilation phenotype where associated with slower aortic growth, ${ }^{19}$ in agreement with our results. 
Our previous cross-sectional study focused exclusively on aortic morphology suggested that patients with reCoA tended to present increased aortic diameters compared with CoA patients with successful repair and no residual narrowing, ${ }^{18}$ yet current results did not reveal an association between reCoA and aortic growth rate. This may be partly due to a relatively short time window, nevertheless significant increases in aortic size were observed in other patients over this time. Aortic stenosis and regurgitation, as well as history of hypertension, were also not associated with aortic growth rate. It has been previously reported in the literature that BAV is a significant predictor of AAo complications in patients with CoA irrespectively of high blood pressure, and aortic dilation and dissection in CoA patients are not only explained by hypertension and valve dysfunction. ${ }^{13}$ Although BAV and CoA have been characterised as 'two villainous cardiovascular lesions in cahoots' with a similar pathophysiology that is part of a diffuse arteriopathy, ${ }^{26}$ yet the heterogeneity and abnormal haemodynamics characterising BAV disease make it difficult to identify the key mechanisms that should be investigated through more studies in BAV with and without CoA. 23

This study focused exclusively on patients with BAV. Aortic growth in BAV patients is considerably more prevalent and faster compared with tricuspid aortic valve patients. Current, joint guidelines of the American College of Cardiology/American Heart Association and collaborating societies recommend elective repair for patients with/without BAV when the aortic diameter reaches $\geq 55 \mathrm{~mm}$, or at $50 \mathrm{~mm}$ for those with BAV and: uncontrolled hypertension, family history of dissection and rapid growth rate $>5 \mathrm{~mm} /$ year. The last indication includes presence of severe valve dysfunction (either stenosis/regurgitation) and an aortic diameter $>45 \mathrm{~mm} .{ }^{28}$ Therefore, given that growth rate drives surgical indication in BAV patients, refining the knowledge of its behaviour in association with CoA is warranted. Our study shows that the coexistence of these two entities does not necessarily imply a faster growth rate. As the matter of fact, the presence of CoA showed a slower growth rate despite associated risk factors such as history of hypertension.

Considering the indexed areas and the follow-up measurements from the current study, four patients in this population would be identified to be at risk of aortic dissection and requiring intervention, with area $>10 \mathrm{~cm}^{2} / \mathrm{m}$ and $48-50 \mathrm{~mm}$ diameter. ${ }^{21}{ }^{29}$ It should also be noted that our population is relatively young (average age 39 years overall) and while we cannot extrapolate information on growth rate beyond the time window that was studied, we cannot exclude further aortic dilation in these patients. This requires further analysis, not only by considering a wider timeframe, but potentially also making use of predictive modelling methodologies that may allow us to study aortic growth over time.

Finally, measurements were performed during both systole and diastole. While this may not reflect clinical practice, it beings to address the potentially dynamic nature of the problem, which should be addressed in the future in a separate study focusing on changes in aortic distensibility.

The study has the limitations of a retrospective design. Blood pressure data (actual cuff pressure at the time of CMR) and other risk factors (such as smoke and dyslipidaemia) were not available for all patients based on the available clinical data; however, history of hypertension was considered in the analysis. With regards to dimensional assessment, admittedly CMR imaging offers a limited spatial resolution compared with echocardiography and CT imaging, nevertheless our results agree with values of growth rate reported in the literature from echocardiographic studies. ${ }^{19}$ While TTE remains the technique of choice in the clinical follow-up of BAV patients, a comparison between TTE and CMR in measuring proximal AAo diameters in BAV patients showed that TTE measurements may be inaccurate in the presence of root asymmetry and RN fusion pattern. ${ }^{30} \mathrm{CMR}$ imaging is increasingly utilised in clinical follow-up and assessment of these patients, particularly when aortic morphology cannot be accurately assessed by TTE. Also, in the case of aortic dilatation or aneurysmal enlargement, a CMR scan is recommended to fully evaluate the thoracic aorta, in terms of further assessing the severity of dilation and the involvement of the AAo. ${ }^{17}$

This study highlights the importance of studying aortic growth in BAV patients with and without associated CoA, since BAV-aortopathy is likely a reflection of BAV morphotype rather than CoA or its physiological effects. In this light, CoA patients with concomitant BAV disease should be considered as a separate group having two different disease phenotypes and receiving different monitoring and treatment from isolated CoA patients. In addition, this study confirms that patients with isolated BAV and $\mathrm{RN}$ fusion pattern may present substantially faster aortic root growth, whereas the more common aortic dilation phenotype and valve fusion pattern appeared as a more stable disease entity with slower progression. Therefore, patient stratification and identification of risk factors of aortic growth can lead to a more targeted prognosis and monitoring of the condition.

\section{Twitter Graham Stuart @ag_stuart}

Acknowledgements The authors acknowledge generous support from the British Heart Foundation.

Contributors FS, MC, CBD and GB planned the design of the study. FS, BB, MVO, MB, EGM and VDF collected and analysed MRI data. FS, BB, MVO, GS, MC, $\mathrm{CBD}$ and $\mathrm{GB}$ contributed to data analysis and interpretation. FS and GB draft the manuscript and all authors critically revised the manuscript and contributed to the final version.

Funding $\mathrm{CBD}$ and this study are supported by the Bristol National Institute of Health Research (NIHR) Biomedical Research Centre (BRC).

Disclaimer The views expressed are those of the authors and not necessarily those of the National Health Service, NIHR or Department of Health and Social Care.

Competing interests CBD is a consultant for Circle Cardiovascular Imaging (Calgary, Canada).

Patient consent for publication Not required. 
Ethics approval All datasets were anonymised and, in view of the retrospective study design, formal ethical approval was waived by the local Institutional Research and Innovation Department in view of the retrospective nature of the study.

Provenance and peer review Not commissioned; externally peer reviewed.

Data availability statement All data relevant to the study are included in the article or uploaded as supplementary information.

Open access This is an open access article distributed in accordance with the Creative Commons Attribution 4.0 Unported (CC BY 4.0) license, which permits others to copy, redistribute, remix, transform and build upon this work for any purpose, provided the original work is properly cited, a link to the licence is given, and indication of whether changes were made. See: https://creativecommons.org/ licenses/by/4.0/.

\section{ORCID iDs}

Bostjan Berlot http://orcid.org/0000-0002-5805-5368

Giovanni Biglino http://orcid.org/0000-0003-0413-149X

\section{REFERENCES}

1 Ward C. Clinical significance of the bicuspid aortic valve. Heart 2000;83:81-5.

2 Russo CF, Mazzetti S, Garatti A, et al. Aortic complications after bicuspid aortic valve replacement: long-term results. Ann Thorac Surg 2002;74:S1773-6.

3 Michelena HI, Khanna AD, Mahoney D, et al. Incidence of aortic complications in patients with bicuspid aortic valves. JAMA 2011;306:1104-12.

4 Yang P, Schmit BM, Fu C, et al. Smooth muscle cell-specific TGFBR1 deficiency promotes aortic aneurysm formation by stimulating multiple signaling events. Sci Rep 2016;6.

5 Ignatieva E, Kostina D, Irtyuga $\mathrm{O}$, et al. Mechanisms of smooth muscle cell differentiation are distinctly altered in thoracic aortic aneurysms associated with bicuspid or tricuspid aortic valves. Front Physiol 2017;8:536.

6 Prapa M, Ho SY, Yen Ho S. Risk stratification in bicuspid aortic valve disease: still more work to do. Eur J Cardiothorac Surg 2012;41:327-8.

7 Siu SC, Silversides CK. Bicuspid aortic valve disease. J Am Coll Cardiol 2010;55:2789-800.

8 Thanassoulis G, Yip JWL, Filion K, et al. Retrospective study to identify predictors of the presence and rapid progression of aortic dilatation in patients with bicuspid aortic valves. Nat Clin Pract Cardiovasc Med 2008;5:821-8.

9 Sievers H-H, Schmidtke C. A classification system for the bicuspid aortic valve from 304 surgical specimens. J Thorac Cardiovasc Surg 2007;133:1226-33.

10 Sievers H-H, Stierle U, Hachmann RMS, et al. New insights in the association between bicuspid aortic valve phenotype, aortic configuration and valve haemodynamics. Eur J Cardiothorac Surg 2016:49:439-46.

11 Della Corte A, Bancone C, Quarto C, et al. Predictors of ascending aortic dilatation with bicuspid aortic valve: a wide spectrum of disease expression. Eur J Cardiothorac Surg 2007;31:397-405. discussion -5 .

12 Losenno KL, Goodman RL, Chu MWA. Bicuspid aortic valve disease and ascending aortic aneurysms: gaps in knowledge. Cardiol Res Pract 2012;2012:145202
13 Oliver JM, Gallego P, Gonzalez A, et al. Risk factors for aortic complications in adults with coarctation of the aorta. J Am Coll Cardiol 2004;44:1641-7.

14 Zhao Q, Shi K, Yang Z-gang, et al. Predictors of aortic dilation in patients with coarctation of the aorta: evaluation with dual-source computed tomography. BMC Cardiovasc Disord 2018;18.

15 Clair M, Fernandes SM, Khairy P, et al. Aortic valve dysfunction and aortic dilation in adults with coarctation of the aorta. Congenit Heart Dis 2014;9:235-43.

16 Frandsen EL, Burchill LJ, Khan AM, et al. Ascending aortic size in aortic coarctation depends on aortic valve morphology: understanding the bicuspid valve phenotype. Int $J$ Cardiol 2018:250:106-9.

17 Braverman AC. Aortic involvement in patients with a bicuspid aortic valve. Heart 2011;97:506-13.

18 Sophocleous F, Biffi B, Milano EG, et al. Aortic morphological variability in patients with bicuspid aortic valve and aortic coarctation. Eur J Cardiothorac Surg 2019;55:704-13.

19 Della Corte A, Bancone C, Buonocore M, et al. Pattern of ascending aortic dimensions predicts the growth rate of the aorta in patients with bicuspid aortic valve. JACC Cardiovasc Imaging 2013;6:1301-10.

20 Baumgartner H, Falk V, Bax JJ, et al. 2017 ESC/EACTS quidelines for the management of valvular heart disease. Eur Heart $J$ 2017;38:2739-91.

21 Svensson LG, Kim K-H, Lytle BW, et al. Relationship of aortic cross-sectional area to height ratio and the risk of aortic dissection in patients with bicuspid aortic valves. J Thorac Cardiovasc Surg 2003;126:892-3.

22 Fazel SS, Mallidi HR, Lee RS, et al. The aortopathy of bicuspid aortic valve disease has distinctive patterns and usually involves the transverse aortic arch. J Thorac Cardiovasc Surg 2008;135:901-7.

23 Oliver JM, Alonso-Gonzalez R, Gonzalez AE, et al. Risk of aortic root or ascending aorta complications in patients with bicuspid aortic valve with and without coarctation of the aorta. Am J Cardiol 2009;104:1001-6.

24 Beroukhim RS, Kruzick TL, Taylor AL, et al. Progression of aortic dilation in children with a functionally normal bicuspid aortic valve. Am J Cardiol 2006;98:828-30.

25 Avadhani SA, Martin-Doyle W, Shaikh AY, et al. Predictors of ascending aortic dilation in bicuspid aortic valve disease: a five-year prospective study. Am J Med 2015;128:647-52.

26 Warnes CA. Bicuspid aortic valve and coarctation: two villains part of a diffuse problem. Heart 2003;89:965-6.

27 Fedak PWM. Bicuspid aortic valve syndrome: heterogeneous but predictable? Eur Heart J 2008;29:432-3.

28 Hiratzka LF, Creager MA, Isselbacher EM, et al. Surgery for aortic dilatation in patients with bicuspid aortic valves. J Am Coll Cardiol 2016;67:724-31.

29 Masri A, Kalahasti V, Svensson LG, et al. Aortic cross-sectional Area/Height ratio and outcomes in patients with bicuspid aortic valve and a dilated ascending aorta. Circ Cardiovasc Imaging 2017; 10:e006249.

30 Vis JC, Rodríguez-Palomares JF, Teixidó-Tura G, et al. Implications of asymmetry and valvular morphotype on echocardiographic measurements of the aortic root in bicuspid aortic valve. J Am Soc Echocardiogr 2019;32:105-12. 